\title{
Mitochondrial DNA haplogroup $M$ is associated with late onset of hepatocellular carcinoma
}

\author{
ZHANJUN GUO ${ }^{1}$, HUA YANG ${ }^{1}$, CUIJU WANG ${ }^{2,3}$ and SHUFENG LIU ${ }^{3}$ \\ Departments of ${ }^{1}$ Gastroenterology and Hepatology, and ${ }^{2}$ Gynaecology Ultrasound, \\ The Fourth Hospital of Hebei Medical University; ${ }^{3}$ Hebei Key Lab of Laboratory Animal Science, \\ Hebei Medical University, Shijiazhuang, Hebei 050011, P.R. China
}

Received September 23, 2011; Accepted November 18, 2011

DOI: $10.3892 /$ etm.2011.434

\begin{abstract}
The accumulation of single nucleotide polymorphisms (SNPs) in the displacement loop (D-loop) of mitochondrial DNA (mtDNA) has been associated with various types of cancer. The association of SNPs with cancer risk and disease outcome has been exhaustively studied. In this study, we investigated the association of age-at-onset and SNPs in the mitochondrial D-loop using a population-based series of hepatocellular carcinoma (HCC) patients. Haplogroup M (489C) and allele 235G were identified for their association with the late onset of HCC by the log-rank test. In an overall multivariate analysis, haplogroup M (489C) was identified as an independent predictive factor for the age-at-onset of HCC at borderline significant levels [relative risk, 1.736; 95\% confidence interval (CI), 0.967-3.115; p=0.065]. Genetic polymorphisms in the D-loop are predictive markers for age-at-onset in HCC patients. Accordingly, the analysis of genetic polymorphisms in the mitochondrial D-loop may help to identify HCC patient subgroups at high risk of early onset of the disease.
\end{abstract}

\section{Introduction}

Hepatocellular carcinoma (HCC) is the fifth most frequent type of cancer and the third leading cause of cancer mortality worldwide, with over half a million cases of mortality every year (1). $\mathrm{HCC}$ is also common in China. According to the annual cancer incidence and mortality report, the incidence and mortality rates of HCC in China over the last decade were 300,000 and 306,000 cases, respectively $(2,3)$. This disease is strongly associated with several risk factors, including chronic hepatitis $\mathrm{B}$ virus (HBV) and chronic hepatitis $\mathrm{C}$ virus (HCV) infection, as well as alcohol abuse (4). Certain epidemic factors have been

Correspondence to: Dr Cuiju Wang, Department of Gynaecology Ultrasound, The Fourth Hospital of Hebei Medical University, 12 Jiankang Road, Shijiazhuang, Hebei 050011, P.R. China E-mail: irenguo@yahoo.cn

Key words: hepatocellular carcinoma, displacement loop, single nucleotide polymorphism, age-at-onset identified as risk factors or outcome predictors for HCC (5-7); however, the true mechanism of this cancer remains unknown. To date, few studies have focused on the genetic factors associated with age-at-onset of this cancer, although they have demonstrated the genetic prevalence of this disease (8).

Hepatitis virus infection and alcohol abuse are associated with increased oxidative stress in liver cells, resulting in DNA changes including mitochondrial DNA (mtDNA) instability $(9,10)$. The human mitochondrial genome is $16 \mathrm{~kb}$ in length and is a closed-circular duplex molecule that contains 37 genes, including two ribosomal RNAs and complete sets of 22 transfer RNAs (tRNAs) (11). mtDNA is believed to be more susceptible to DNA damage and acquires mutations at a higher rate than nuclear DNA owing to high levels of reactive oxygen species (ROS), lack of protective histones and limited capacity for DNA repair in the mitochondria (12-14). Thus, somatic mtDNA mutations occur in a wide variety of degenerative diseases and cancers $(15,16)$ and may be homoplasmic by clonal expansion $(17,18)$ or heteroplasmic in tumor tissues $(19,20)$. In a number of cancers, including hepatitis virus-related $\mathrm{HCC}$, somatic mutations are frequently located in the mtDNA non-coding region, termed the displacement loop (D-loop) $(21,22)$. This region is important for regulating the replication and expression of the mitochondrial genome since it contains the leading-strand origin of replication and is the main promoter for transcription (23).

We sequenced the D-loop that contains a length of 1,122 bps (nucleotides 16024-16569 and 1-576; www.mitomap. org) in the blood from HCC patients and identified 92 single nucleotide polymorphims (SNPs) in the D-loop. We also identified cancer risk and outcome associated SNPs $(24,25)$. In the present study, we assess the correlation between germline SNPs of the D-loop and age-at-onset in HCC patients.

\section{Materials and methods}

Tissue specimens and DNA extraction. Blood samples were collected at the Fourth Hospital of Hebei Medical University (China) from $60 \mathrm{HCC}$ patients who underwent $\mathrm{HCC}$ resection in the Department of Hepatobiliary Surgery between 2007 and 2008. All patients originated from the Hebei Province of China, a high-risk area for HCC. Whole blood was obtained from corresponding HCC patients. Mitochondria isolation 
and mtDNA extraction were carried out using the Blood Mitochondrial DNA Extraction Kit (Genmed Scientific Inc., Shanghai, China). The study was approved by the Human Tissue Research Committee of the Fourth Hospital of Hebei Medical University. All patients provided written informed consent for the collection of samples and subsequent analysis.

Polymerase chain reaction (PCR) amplification and sequence analysis. The forward primer, 5'-CCCCATGCTTACAAGCAA GT-3' (nucleotide 16190-16209); and reverse primer, 5'-GCTTT GAGGAGGTAAGCTAC-3' (nucleotide 602-583) were used for the amplification of a 982-bp product from the mtDNA D-loop region as previously described (15). PCR was performed according to the protocol of the PCR Master Mix Kit (Promega, Madison, WI, USA) and purified prior to sequencing. Cycle sequencing was carried out using the Dye Terminator Cycle Sequencing Ready Reaction Kit (Applied Biosystems, Foster City, CA, USA) and the products were then separated on the ABI PRISM Genetic Analyzer 3100 (Applied Biosystems). Polymorphisms were confirmed by repeated analyses from the two strands. SNPs were identified directly from blood mitochondria.

Statistical analysis. The age-at-onset curve of the HCC patients was calculated using the Kaplan-Meier method at each SNP site, and compared using the log-rank test. Multivariate survival analysis was performed using a Cox proportional hazards model. The statistical analyses were carried out using the SPSS 11.5 software package (SPSS Company, Chicago, IL, USA). A p-value of $<0.05$ was considered to indicate statistically significant differences.

\section{Results}

A total of 60 patients, including $49 \mathrm{HBV}$-associated and 11 alcohol-associated HCC patients, were enrolled in this study. The age-at-onset distribution of HCC patients is listed in Table I. Those analyzed included 6 patients aged $<40$ years, 12 patients aged 40-50, 30 patients aged 51-60 and 12 patients aged $>60$. None of these patients had received any adjuvant chemotherapy or radiation therapy following $\mathrm{HCC}$ resection. The age-at-onset and clinical characteristics of the HCC patients were analyzed using the Kaplan-Meier method and were compared using the log-rank test. Gender, portal vein thrombosis, child classification and tumor quantity were not associated with age-at-onset according to the results of the log-rank test. However, TNM classification and tumor size correlated with age-at-onset at statistically significant levels (Table II).

Subsequently, the correlation between mtDNA genotype and age-at-onset was compared. We exluded the SNPs with a minor allele frequency of $<5 \%$ and obtained 21 SNPs for further analysis. The HCC patients were divided into two groups on the basis of their genotype at each SNP site, and the age-at-onset curve was plotted using the Kaplan-Meier method for all HCC patients at these sites. A dramatic difference in age-at-onset appeared at sites 489 and 235 as shown by the log-rank test (Fig. 1). The 489C genotype, known as mtDNA haplogroup $M$, was significantly associated with a late age-at-onset of HCC when compared with halpogroup N $(p=0.015)$ (Fig. 1). Another age-at-onset-associated SNP was
Table I. Age-at-onset distribution in ESCC patients.

\begin{tabular}{lcc}
\hline \multirow{2}{*}{$\begin{array}{c}\text { Age } \\
\text { (years) }\end{array}$} & \multicolumn{2}{c}{ No. of cases } \\
\cline { 2 - 3 } & Male & Female \\
\hline 40 & 4 & 2 \\
$41-50$ & 11 & 1 \\
$51-60$ & 28 & 2 \\
$>60$ & 10 & 2 \\
\hline
\end{tabular}

ESCC, esophageal squamous cell carcinoma.

Table II. Clinical characteristics and their association with age-at-onset in $\mathrm{HCC}$ patients.

\begin{tabular}{lcc}
\hline Characteristics & No. of cases & p-value \\
\hline Gender & 53 & 0.370 \\
Male & 7 & \\
Female & & 0.062 \\
TNM classification & 15 & \\
I & 45 & 0.405 \\
II & & \\
Portal vein thrombosis & 10 & 0.122 \\
Yes & 50 & \\
No & & \\
Child classification & 57 & \\
A & 3 & \\
B & & \\
Tumor size (diameter) & 10 & \\
$<5$ cm & 50 & \\
$\geq 5$ cm & & \\
Tumor quantity & 50 & \\
Single & 10 & \\
Multiple & & \\
\hline
\end{tabular}

HCC, hepatocellular carcinoma.

identified at site 235 with frequency allele $235 \mathrm{G}$ and was linked with the late onset of HCC $(\mathrm{p}=0.009)$.

Using the Cox proportional hazards model, we performed multivariate analysis of HCC age-at-onset predictors including TNM classification, tumor size and the two SNPs. As shown in Table III, haplogroup M (489C) was identified as an independent predictive factor for age-at-onset of HCC at borderline significant levels [relative risk, 1.736; $95 \%$ confidence interval (CI), 0.967-3.115; p=0.065].

\section{Discussion}

Selected SNPs in the D-loop region have previously been examined for their ability to predict cancer risk and outcome in many types of cancer (26-28). The present study has extended these analyses to determine the relationships between 
A

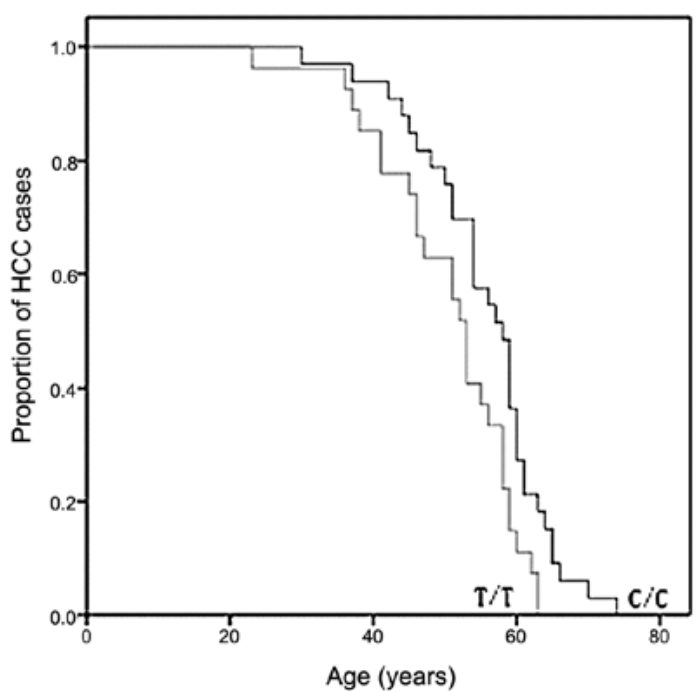

B

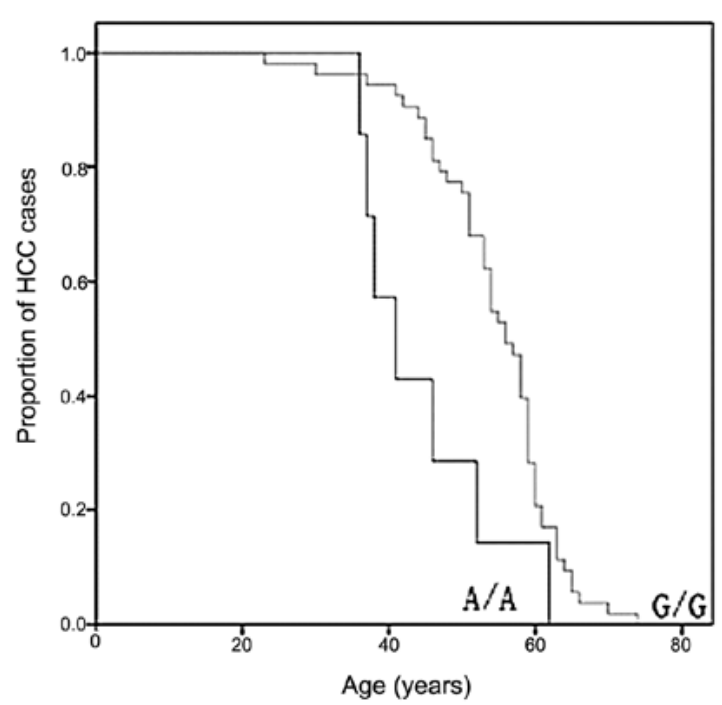

Figure 1. Comparison of the assocation between the age-at-onset of HCC and the (A) 489 and (B) 235 sites in the D-loop. D-loop, displacement loop; HCC, hepatocellular carcinoma.

Table III. Multivariate analysis of predictive factors associated with the age-at-onset of HCC.

\begin{tabular}{lccc}
\hline Factors & Relative risk & $95 \%$ CI & p-value \\
\hline Tumor size & 2.023 & $0.831-4.926$ & 0.121 \\
TNM classification & 1.179 & $0.573-2.427$ & 0.654 \\
$235(\mathrm{G} / \mathrm{A})$ & 0.611 & $0.254-1.470$ & 0.271 \\
$489(\mathrm{C} / \mathrm{T})$ & 1.736 & $0.967-3.115$ & 0.065 \\
\hline
\end{tabular}

HCC, hepatocellular carcinoma; CI, confidence interval.

age-at-onset and germline SNPs in a continuous sequence of mtDNA between nucleotides 16190 and 583 in HCC patients. The SNPsm, 489C/T and 235G/A, were identified for their association with age-at-onset at statistically significant levels by the log-rank test. In an overall multivariate analysis, haplogroup M
(489C) was identified as an independent predictive factor for age-at-onset of HCC at borderline significant levels. The results from the log-rank test revealed that disease advancement with a larger tumor size and a more serious clinical stage was more prevalent in younger patients. However, this was not shown in the multivariate analysis.

We have previously performed a number of studies on D-loop SNPs as predictive factors for digestive tract cancers $(24,25,29)$. In the present study, for the first time, we suggest that, other than as predictors for cancer risk and outcome, SNPs in the D-loop are also predictors for age-at-onset in HCC patients. All non-African lineages belong to two founder clusters, namely haplogroups $\mathrm{M}$ and $\mathrm{N} ; 489 \mathrm{C}$, defined as haplogroup $\mathrm{M}$, and 489T, defined as haplogroup N. According to the 'out of Africa' theory, these are both derived from the L3 mtDNA African lineage (30). The functional significance of the mtDNA haplogroups and their association with human behaviors requires further study, although certain haplogroups have been identified as markers for special diseases $(31,32)$. The results from this study require validation in the form of larger population sizes and laboratory-based functional studies.

The D-loop region of mtDNA is crucial for the regulation of mitochondrial genome replication and expression. SNPs in this region might affect mtDNA replication and lead to the alteration of the electron transport chain, which is responsible for the release of highly reactive oxygen species (ROS) and could contribute to nuclear genome damage as well as cancer initiation and promotion (33-35). These two SNPs may alter the transcription of the mitochondrial genome, and this may subsequently enhance the production of ROS when the mitochondrial transcription is altered (36). These ROS-mediated mechanisms may thereby accelerate tumor development.

In conclusion, SNPs in the D-loop have been found to be biomarkers for the age-at-onset of HCC. The analysis of genetic polymorphisms in the D-loop might help to identify patient subgroups at high risk for an early onset, thereby helping to refine therapeutic decisions in HCC cancers.

\section{Acknowledgements}

This study was supported by the National Natural Science Foundation of China (No. 30801384). The research was supported in part by the Natural Science Foundation of Hebei Province (No. C2008000958).

\section{References}

1. Gomaa AI, Khan SA, Toledano MB, Waked I and Taylor-Robinson SD: hepatocellular carcinoma: Epidemiology, risk factors and pathogenesis. World J Gastroenterol 14: 4300-4308, 2008.

2. Sun Z, Ming L, Zhu X and Lu J: Prevetion and control of hepatitis B in China. J Med Virol 67: 447-450, 2002.

3. Ferlay J, Bray F, Pisani P and Parkin DM (eds): Globocan 2000: Cancer Incidence, Mortality and Prevalence Worldwide. Version 1.0. IARC Press, Lyon, 2001.

4. Caldwell S and Park SH: The epidemiology of hepatocellular cancer: from the perspectives of public health problem to tumor biology. J Gastroenterol 44: 96-101, 2009.

5. Maki A, Kono H, Gupta M, Asakawa M, Suzuki T, Matsuda M, Fujii $\mathrm{H}$ and Rusyn I: Predictive power of biomarkers of oxidative stress and inflammation in patients with hepatitis $\mathrm{C}$ virus-associated hepatocellular carcinoma. Anal Surg Oncol 14: 1182-1190, 2007. 
6. Okada S, Shimada K, Yamamoto J, Takayama T, Kosuge T, Yamasaki S, Sakamoto M and Hirohashi S: Predictive factors for postoperative recurrence of hepatocellular carcinoma. Gastroenterology 106: 1618-1624, 1994.

7. Minagawa M,Makuuchi M, Takayama T and Kokudo N: Selection criteria for repeat hepatectomy in patients with recurrent hepatocellular carcinoma. Ann Surg 238: 703-710, 2003.

8. Wong N, Yeo W, Wong WL, Wong NL, Chan KY, Mo FK, Koh J, Chan SL, Chan AT, Lai PB, et al: TOP2A overexpression in hepatocellular carcinoma correlates with early age onset, shorter patients survival and chemoresistance. Int J Cancer 124 644-652, 2009.

9. Schwarz KB: Oxidative stress during viral infection: a review. Free Radical Biol Med 21: 641-649, 1996.

10. Mansouri A, Fromenty B, Berson A, Robin MA, Grimbert S, Beaugrand M, Erlingr S and Pessayre D: Multiple hepatic mitochondrial DNA deletions suggest premature oxidative aging in alcoholic patients. J Hepatol 27: 96-102, 1997.

11. Shadel GS and Clayton DA: Mitochondrial DNA maintenance in vertebrates. Annu Rev Biochem 66: 409-435, 1997.

12. DiMauro S and Schon EA: Mitochondrial DNA mutations in human disease. Am J Med Genet 106: 18-26, 2001.

13. Beal MF: Mitochondia, free radicals, and neurodegeneration. Curr Opin Neurobiol 6: 661-666, 1996.

14. Lightowlers RN, Chinnery PF, Turnbull DM, and Howell N: Mammalian mitochondrial genetics: heredity, heteroplasmy and disease. Trends Genet 13: 450-455, 1997.

15. Wallace DC: Mouse models for mitochondrial disease. Am J Med Genet 106: 71-93, 2001

16. Fliss MS, Usadel H, Caballero OL, Wu L, Buta MR, Eleff SM, Jen J and Sidransky D: Facile detection of mitochondrial DNA mutations in tumors and bodily fluids. Science 287: 2017-2019, 2000.

17. Nomoto S, Yamashita K, Koshikawa K, Nakao A and Sidransky D: Mitochondrial D-loop mutation as clonal markers in multicentric hepatocellular carcimona and plasma. Clin Cancer Res 8: 481-487, 2002.

18. Mambo E, Gao X, Cohen Y, Guo Z, Talalay P and Sidransky D: Electrophile and oxidant damage of mitochondrial DNA leading to rapid evolution of homoplasmic mutations. Proc Natl Acad Sci USA 100: 1838-1843, 2003.

19. Yoneyama H, Hara T, Kato Y, Yamori T, Matsuura ET and Koike K: Nucleotide sequence variation is frequently in the mitochondrial DNA displacement loop region of individual human tumor cells. Mol Cancer Res 3: 14-20, 2005.

20. Jakupciak JP, Maragh S, Markowitz ME, Greenberg AK, Hoque MO, Maitra A, Barker PE, Wagner PD, Rom WN Srivastava S, Sidransky D and O'Connell CD: Performance of mitochondrial DNA mutations detecting early stage cancer. BMC Cancer 8: 285, 2008

21. Nashikawa M, Nishiguchi S, Shiomi S, Tamori A, Koh N, Takeda T, Kubo S, Hirohashi K, Kinoshita H, Sato E and Inoue M: Somatic mutation of mitochondrial DNA in cancerous and noncancerous liver tissue in individuals with hepatocellular carcinoma. Cancer Res 61: 1843-1845, 2001.

22. Sanchez-Cespedes M, Parrella P, Nomoto S, Cohen D, Xiao Y, Esteller M, Jeronimo C, Jordan RC, Nicol T, Koch WM, Schoenberg M, Mazzarelli P, Fazio VM and Sidransky D: Identification of a mononucleotide repeat as a major target for mitochondrial DNA alterations in human tumors. Cancer Res 61 : 7015-7019, 2001
23. Taanman JW: The mitochondrial genome: structure, transcription, translation and replication. Biochim Biophys Acta 1410: 103-123, 1999.

24. Zhang R, Zhang F, Wang C, Wang S, Shiao Y-H and Guo Z: Identification of sequence polymorphism in the D-loop region of mitochondria DNA as a risk factor for hepatocellular carcinoma with distinct etiology. J Exp Clin Cancer Res 29: 130, 2010.

25. Wang C, Zhang F, Fan H, Peng L, Zhang R, Liu S and Guo Z: Sequence polymorphisms of mitochondrial D-loop and hepatocellular carcinoma outcome. Biochem Biophys Res Commun 406: 493-496, 2011

26. Navaglia F, Basso D, Fogar P, Sperti C, Greco E, Zambon CF, Stranges A, Falda A, Pizzi S, Parenti A, Pedrazzoli S and Plebani M: Mitochondrial DNA D-loop in pancreatic cancer: somatic mutations are epiphenomena while the germline $16519 \mathrm{~T}$ variant worsens metabolism and outcome. Am J Clin Pathol 126: 593-601, 2006.

27. Wang L, Bamlet WR, de Andrade M, Boardman LA, Cunningham JM, Thibodeau SN and Petersen GM: Mitochondrial genetic polymorphisms and pancreatic cancer risk. Cancer Epidemiol Biomarkers Prev 16: 1455-1459, 2007.

28. Wang L, McDonnell SK, Hebbring SJ, Cunningham JM, St Sauver J, Cerhan JR, Isaya G, Schaid DJ and Thibodeau SN: Polymorphisms in mitochondrial genes and prostate cancer risk. Cancer Epidemiol Biomarkers Prev 17: 3558-3566, 2008.

29. Zhang R, Wang R, Zhang F, Wu C, Fan H, li Y, Wang C and Guo Z: Single nucleotide polymorphisms in the mitochondrial displacement loop and outcome of esophageal squamous cell carcinoma. J Exp Clin Cancer Res 29: 155, 2010.

30. González AM, Larruga JM, Abu-Amero KK, Shi Y, Pestano J and Cabrera VM: Mitochondrial lineage M1 traces an early human backflow to Africa. BMC Genomics 8: 223, 2007.

31. Carelli V, Achilli A, Valentino ML, Rengo C, Semino O, Pala M, Olivieri A, Mattiazzi M, Pallotti F, Carrara F, et al: Haplogroup effects and recombination of mitochondrial DNA: novel clues from the analysis of Leber hereditary optic neuropathy pedigrees. Am J Hum Genet 78: 564-574, 2006.

32. Udar N, Atilano SR, Memarzadeh M, Boyer DS, Chwa M, Lu S, Maguen B, Langberg J, Coskun P, Wallace DC, et al: Mitochondrial DNA haplogroups associated with age-related macular degeneration. Invest Ophthalmol Vis Sci 50: 1966-2974, 2009.

33. Bandy B and Davision AJ: Mitochondrial mutations may increase oxidative stress: implications for carcinogenesis and aging? Free Radic Biol Med 8: 523-539, 1990.

34. Gille JJ and Joenje H: Cell culture models for oxidative stress: superoxide and hydrogen peroxide versus normobaric heperoxia. Mutat Res 275: 405-414, 1992.

35. Shigenaga MK, Hagen TM and Ames BN: Oxidative damage and mitochondrial decay in aging. Proc Natl Acad Sci USA 91: 10771-10778, 1994.

36. Dement GA, Maloney SC and Reeves R: Nuclear HMGA1 nonhistone chromatin proteins directly influence mitochondrial transcription, maintenance, and function. Exp Cell Res 313: 77-87, 2007. 